\title{
Introduction: neurogenomics and neuroproteomics
}

\author{
Mitchel S. Berger, M.D., ${ }^{1}$ William T. Couldwell, M.D., Ph.D., ${ }^{2}$ \\ James T. Rutka, M.D., Ph.D., ${ }^{3}$ and Nathan R. Selden, M.D., Ph.D. ${ }^{4}$ \\ ${ }^{1}$ Department of Neurosurgery, University of California, San Francisco, California; \\ ${ }^{2}$ Department of Neurosurgery, University of Utah, Salt Lake City, Utah; ${ }^{3}$ Department of Neurosurgery, \\ Hospital for Sick Children, University of Toronto, Ontario, Canada; and ${ }^{4}$ Department of Neurological \\ Surgery, Oregon Health \& Science University, Portland, Oregon
}

$\mathrm{F}$ Tor this issue of Neurosurgical Focus, we are pleased to present a spectrum of interesting articles covering a wide range of contemporary genomic and proteomic topics specifically related to neurosurgical diseases. The authors review a broad range of contemporary population genetic and molecular techniques available for the study of neurological disease. The issue begins with articles on broadly applied population genetic tools used to identify the relative risk of neurosurgical diseases among the kindred of affected individuals. One such example is the Utah population data bank described by Niazi et al. Subsequent articles describe game-changing contemporary molecular techniques used to provide screening for genetic alterations associated with a specific disease or phenotype. Examples are the genome-wide association studies described by Cowperthwaite et al. and the next-generation screening techniques for genetic alteration applied to medulloblastomas by Taylor et al. A review of the role of miRNAs in brain tumor biology is provided by Kalani et al. Chen and colleagues describe the identification of distinct functional genomic altera- tions among vast genetic changes as a strategy to target tumor cell vulnerabilities with novel therapies. The application of siRNA technology to induce therapeutic functional changes in gene expression in glioblastoma multiforme cells is described in the article by Thaker et al. In the remaining articles the authors discuss genetic and proteomic changes associated with specific diseases, including head trauma (Dardiotis et al.), Parkinson disease (Hadjigeorgiou et al.), and neurofibromatosis Type 1 (Gottfried et al.). Dhandapani et al. discuss their novel proteomic studies in patients with subarachnoid hemorrhage, which provide proof of principle and promise for future development of diagnostic and therapeutic innovations in this emerging and fascinating area of research. Finally, Vannemreddy and colleagues provide data supporting a role for eNOS in the clinical presentation of intraventricular hemorrhage. Collectively, these papers serve as a solid primer in genomics and proteomics for the practicing neurosurgeon and convey some of the most exciting recent molecular science developments relevant to neurosurgical disease. 